\title{
KERNELS IN LATTICE-ORDERED GROUPS
}

\author{
RICHARD D. BYRD AND JUSTIN T. LLOYD
}

\begin{abstract}
It is shown that a lattice-ordered group has a largest representable convex $l$-subgroup and a largest normal valued convex $l$-subgroup. Other kernels are discussed.
\end{abstract}

1. Introduction. Throughout this note $G$ will denote a lattice-ordered group (l-group). In [7, Theorem 1.6] J. Martinez proved that if $G$ is a representable lattice-ordered group, then $G$ had a largest hyperarchimedean (also called epiarchimedean) convex $l$-subgroup (the hyperarchimedean kernel). P. Conrad [5, Theorem 3.5] extended this result to an arbitrary l-group. In [6] G. O. Kenny announced that a representable $l$-group had a largest archimedean convex $l$-subgroup (the archimedean kernel) and R. Redfield [8, Corollary 2.2] extended this result, again by dropping the hypothesis of representability. In this note we prove that if $G$ is an $l$-group, then $G$ has a largest representable convex $l$-subgroup (the representable kernel) and a largest normal valued convex $l$-subgroup (the normal valued kernel).

If $X$ is a subset of $G$, then $[X]$ denotes the subgroup of $G$ generated by $X$, and $\mathcal{C}(G)$ denotes the lattice of convex $l$-subgroups of $G$. The reader is referred to [4] for the standard terminology and results in l-groups.

2. Kernels. An $l$-group is said to be normal valued if each regular subgroup is normal in the convex $l$-subgroup that covers it. If $G$ is representable, then $G$ is normal valued [1, Corollary 3.2]. Let $\mathcal{H}(G)=\{H \mid H$ is a hyperarchimedean convex $l$-subgroup of $G\}, \operatorname{Ar}(G)=\{H \mid H$ is an archimedean convex $l$ subgroup of $G\}, \mathbb{Q}(G)=\{H \mid H$ is an abelian convex $l$-subgroup of $G\}, \mathcal{R}(G)$ $=\{H \mid H$ is a representable convex $l$-subgroup of $G\}$, and $\mathscr{R}(G)=\{H \mid H$ is a normal valued convex $l$-subgroup of $G$. Then $\mathcal{H}(G) \subseteq \operatorname{Ar}(G) \subseteq \mathcal{Q}(G) \subseteq$ $\Re(G) \subseteq \mathscr{T}(G)$ and, as mentioned in the introduction, $\mathscr{H}(G)$ and $\operatorname{Ar}(G)$ are principal ideals of $\mathcal{C}(G)$.

THEOREM 2.1. Let $G$ be an l-group and $C \in \mathcal{e}(G)$.

(i) If $H, J \in \mathbb{Q}(G)$ and $C \subseteq H$, then $C, H+J \in \mathbb{Q}(G)$.

(ii) If $H, J \in \Re(G)$ and $C \subseteq H$, then $H+J=[H \cup J]$ and $C, H+J \in$ $\Re(G)$.

(iii) If $H, J \in \mathfrak{R}(G)$ and $C \subseteq H$, then $H+J=[H \cup J]$ and $C, H+J \in$ $\Re(G)$.

Received by the editors August 1, 1975.

AMS (MOS) subject classifications (1970). Primary 06A55.

Key words and phrases. Hyperarchimedean kernel, archimedean kernel, representable kernel, normal valued kernel, ideal radical, distributive radical, radical. 
Proof. (i) To show that $H+J$ is abelian, it suffices to show that if $h \in H^{+}$and $j \in J^{+}$, then $h+j=j+h$. Now $h \wedge j \in H \cap J$ and, hence, commutes with $h$ and $j$. Since $h \vee j=h-(h \wedge j)+j=j \vee h$, it follows that $h+j=j+h$.

(ii) To prove that $[H \cup J]$ is representable, it suffices to show that each minimal prime subgroup of $[H \cup J]$ is normal in $[H \cup J]$ [1, Theorem 3.1]. If $M$ is a minimal prime subgroup of $[H \cup J$ ], then $H \nsubseteq M$ or $J \nsubseteq M$.

Case 1. $H \nsubseteq M$ and $J \subseteq M$. Then $J \subseteq n(M)$, the normalizer of $M$ in [ $H \cup J$ ]. By [2, Theorem 3.5], $H \cap M$ is a minimal prime subgroup of $H$ and, since $H$ is representable, $H \cap M$ is normal in $H$ [1, Theorem 3.1]. Hence, by [2, Theorem 3.6], $H \subseteq n(M)$ and so $[H \cup J]=n(M)$.

The cases $H \subseteq M$ and $J \nsubseteq M$, and $H \nsubseteq M$ and $J \nsubseteq M$ are similar to Case 1 and are omitted. Trivially, if $C \subseteq H$, then $C \in \Re(G)$. It will be shown in (iii) that $H+J=[H \cup J]$, since $\Re(G) \subseteq \Re(G)$.

(iii) Let $T$ be a regular subgroup of $[H \cup J], T^{*}$ be the convex $l$-subgroup of $[H \cup J]$ that covers $T$, and let $0<t \in T^{*}$ with $t \notin T$. Then $t=h_{1}+j_{1}$ $+\cdots+h_{n}+j_{n}$ where $h_{i} \in H^{+}$and $j_{i} \in J^{+}$(see [4, p. 1.7]). Thus, for some $1 \leqslant k \leqslant n, h_{k} \notin T$ or $j_{k} \notin T$. Without loss of generality, we may assume that $h_{k} \notin T$. Then $H \cap T$ is a regular subgroup of $H, h_{k} \notin H \cap T, H \cap T^{*}$ is the convex $l$-subgroup of $H$ that covers $H \cap T$, and $h_{k} \in H \cap T^{*}$ [2, Theorem 3.5]. Since $H \in \mathfrak{T}(G), H \cap T$ is normal in $H \cap T^{*}$. Hence, by [2, Theorem 3.6], $T$ is normal in $T^{*}$. Therefore, $[H \cup J] \in \mathfrak{R}(G)$. By [9, Theorem 3], $H+J=[H \cup J]$.

If $C \subseteq H$, then $C \in \Re(G)[9$, Corollary 1, p. 342].

Corollary 2.2. If $G$ is an l-group and $\mathscr{X} \in\{\mathbb{Q}(G), \Re(G), \mathscr{R}(G)\}$, then $\cup \mathfrak{X} \in \mathcal{X}$ and so $\mathfrak{X}$ is a principal ideal of $\mathcal{C}(G)$. Moreover, $\cup \mathcal{X}$ is invariant under all l-automorphisms of $G$.

Let $I(G), D(G)$, and $R(G)$ denote the ideal radical, the distributive radical, and the radical of $C$ respectively (see [3] or [4, Chapter 5]), $g(G)=\{H \mid H$ $\in \mathcal{C}(G)$ and $I(H)=\{0\}\}, \mathscr{D}(G)=\{H \mid H \in \mathcal{C}(G)$ and $D(H)=\{0\}\}$, and $\mathrm{Ra}(G)=\{H \mid H \in \mathcal{C}(G)$ and $R(H)=\{0\}\}$. If $H \in \mathcal{C}(G)$ and $\left\{H_{\lambda} \mid \lambda\right.$ $\in \Lambda\} \subseteq \varrho(G)$, then $D(H)=H \cap D(G)$ and $D\left(\left[\cup H_{\lambda}\right]\right)=\left[\cup D\left(H_{\lambda}\right)\right][3$, p. 126]. The ideal radical and the radical have the same properties.

Theorem 2.3. If $G$ is an l-group and $\mathcal{X} \in\{\operatorname{Ra}(G), \mathcal{D}(G), G(G)\}$, then $\cup \mathcal{X} \in \mathcal{X}, \cup \mathcal{X}$ is invariant under all l-automorphisms of $G$, and $\mathfrak{X}$ is a principal ideal of $\mathcal{C}(G)$.

\section{REFERENCES}

1. R. D. Byrd, Complete distributivity in lattice-ordered groups, Pacific J. Math. 20 (1967), 423-432. MR 34 \#7680.

2. - M-polars in lattice-ordered groups, Czechoslovak Math. J. 18 (93) (1968), 230-239. MR 37 \#2651.

3. R. D. Byrd and J. T. Lloyd,Closed subgroups and complete distributivity in lattice-ordered groups, Math. Z. 101 (1967), 123-130. MR 36 \# 1371.

4. P. Conrad, Lattice-ordered groups, Lecture notes, Tulane University, 1970.

5. ——, Epi-archimedean groups, Czechoslovak Math. J. 24 (99) (1974), 192-218. MR 50 \#203. 
6. G. O. Kenny, Archimedean kernel of a representable l-group, Notices Amer. Math. Soc. 21 (1974), A-590-A-591. Abstract \#74T-A243.

7. J. Martinez, Archimedean-like classes of lattice-ordered groups, Trans. Amer. Math. Soc. 186 (1973), 33-49 (1974). M R 48 \# 10940.

8. R. H. Redfield, Archimedean and basic elements in completely distributive lattice-ordered groups (preprint).

9. S. Wolfenstein, Valeurs normales dans un groupe réticulé, Atti Accad. Naz. Lincei Rend. Cl. Sci. Fis. Mat. Natur. (8) 44 (1968), 337-342. MR 38 \#3201.

Department of Mathematics, University of Houston, Houston, TeXas 77004 